\title{
Synthesis of the tetracyclic skeleton of the galanthamine-type Amaryllidaceae alkaloids
}

\author{
Klára Herke, ${ }^{a}$ László Hazai, ${ }^{\text {b }}$ Máté Szabolcs Hudák, ${ }^{a}$ Janka Ábrahám, ${ }^{\text {a } Z s u z s a n n a ~ S a ́ n t a, ~}$ \\ Viktor Háda, ${ }^{\mathrm{c}}$ Csaba Szántay, Jr., ${ }^{\mathrm{c}}$ and Csaba Szántay ${ }^{\mathrm{a}, \mathrm{b}}$ * \\ ${ }^{a}$ Department of Organic Chemistry and Technology, University of Technology and Economics, \\ Budapest, Hungary \\ ${ }^{b}$ Research Group for Alkaloid Chemistry of the Hungarian Academy of Sciences, H-1111 \\ Budapest, Gellért tér 4., Hungary \\ ${ }^{c}$ Spectroscopic Research Division, Gedeon Richter Plc., H-1475 Budapest 10, P. O. Box 27, \\ Hungary \\ E-mail:szantay@mail.bme.hu
}

\begin{abstract}
The hexahydrobenzofurobenzazepine tetracycle characteristic of the principal galanthamine-type Amaryllidaceae alkaloids was synthesized from a methoxy substituted 2-tetralone via simple and convenient reaction steps.
\end{abstract}

Keywords: Amaryllidaceae alkaloids, galanthamine, tetracycle, benzofurobenzazepine

\section{Introduction}

(-)-Galanthamine (1a) and (-)-lycoramine (2a), alkaloids isolated from the bulbs of various species of the Amaryllidaceae family, contain a saturated benzofurobenzazepine tetracyclic skeleton consisting of four rings $A-D$.

Galanthamine has an important role in the treatment of Alzheimer's disease as an acetylcholinesterase inhibitor $^{1}$ as well as a modulator of neuronal nicotinic receptors. Lycoramine has similar, but less potent activity. A number of total syntheses for galanthamine ${ }^{2,3}$ and lycoramine ${ }^{3-7}$ have been developed. These methods employ various approaches to building up the C-8a quaternary spiro carbon atom and the $A-D$ rings. However, only few data can be found in the literature ${ }^{8}$ on the chemistry and pharmacology of their demethoxy derivatives $\mathbf{1 b}$ and $\mathbf{2 b}$. Recently ${ }^{9}$ we established an efficient synthesis of benzazepines spiro-substituted with a cyclohexenone ring starting from an unsubstituted 2-tetralone. In the present paper we report an extension of that method for methoxy-substituted 2-tetralones according to the retrosynthetic route shown in Scheme 1. 


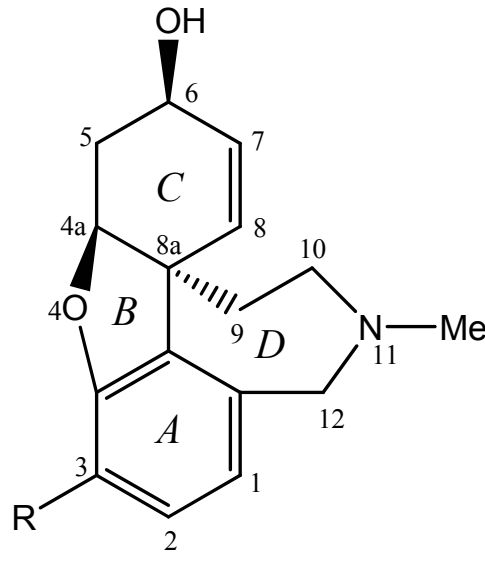

Galanthamine, 1a; $\mathrm{R}=\mathrm{OMe}$

Demethoxygalanthamine, $\mathbf{1 b} ; \mathrm{R}=\mathrm{H}$

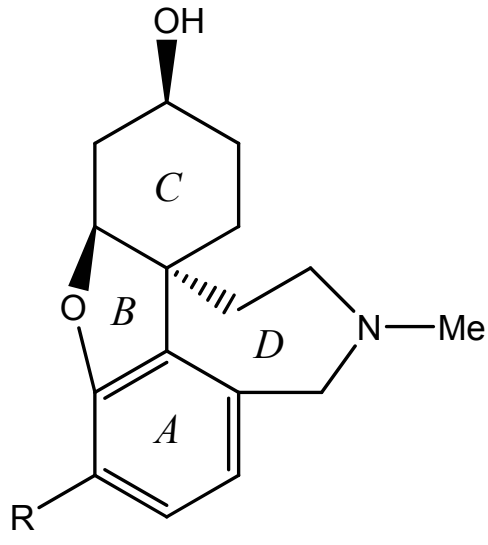

Lycoramine, 2a; $\mathrm{R}=\mathrm{OMe}$

Demethoxylycoramine, $\mathbf{2} \mathbf{b} ; \mathrm{R}=\mathrm{H}$

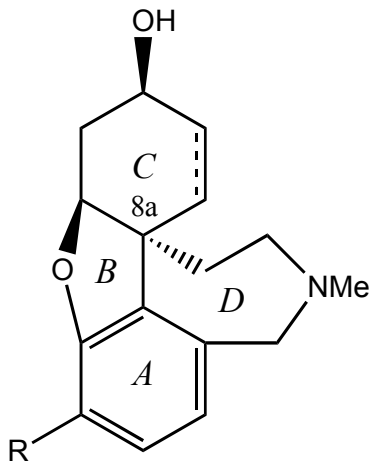

1a,b

$\mathbf{2 a}, \mathbf{b}$<smiles>[R]c1ccc2c(c1OC)C(CCN(C)C)(CC(C)(C)C)CC2</smiles>

3

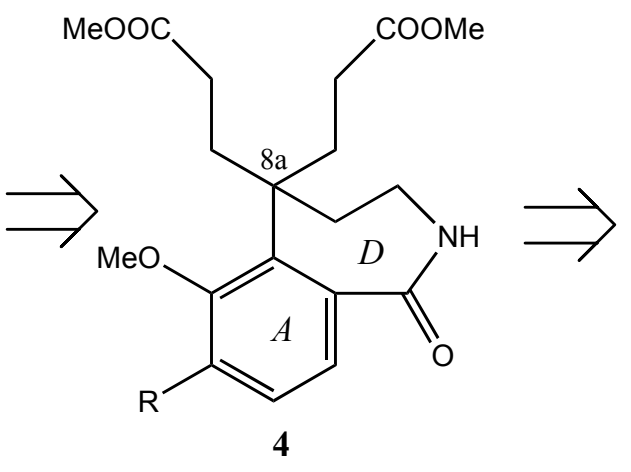

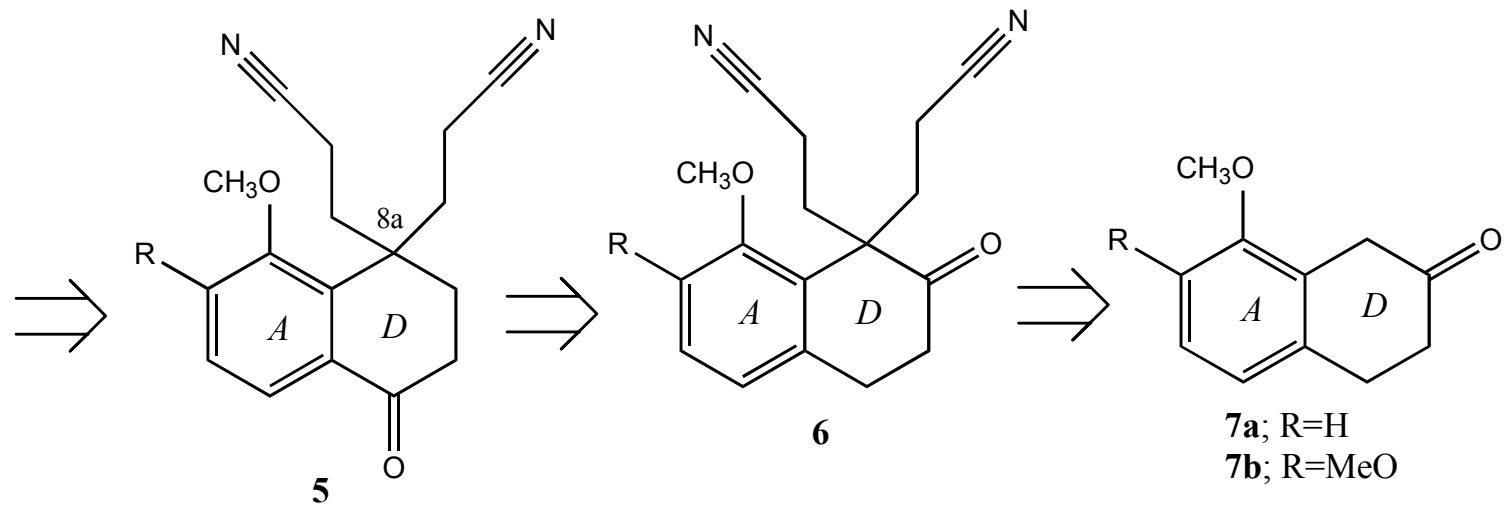

\section{Scheme 1}

The object of this work is the synthesis of the hexahydrobenzofurobenzazepine tetracycle characterising the galanthamine-type alkaloids. Our synthetic method started from 8-methoxy-2tetralone and used simple reaction steps and inexpensive reagents. The $\mathrm{C}-8 \mathrm{a}$ spiro carbon atom was produced via a Dieckmann condensation. 


\section{Results and Discussion}

The starting material 8-methoxy-2-tetralone (7a) is not commercially available, but can be readily prepared from 1,7-dihydroxynaphthalene (8) using known methods (Scheme 2). ${ }^{10,11}$ Compound 8 was methylated with dimethyl sulfate under alkaline conditions $(93 \%)$ and the Birch reduction of the dimethoxy derivative 9 gave ketone $7 \mathbf{a}$ in a good yield (86\%).

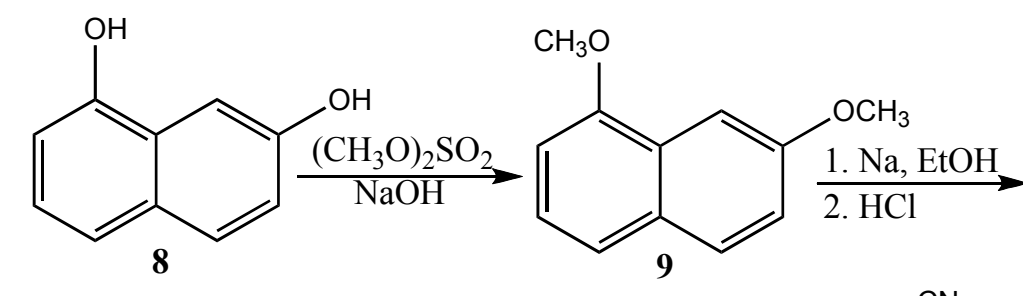<smiles>[R]C1([R])Cc2cccc(OC)c2C(CCC#N)(CCC#N)C1=O</smiles>

10; $\mathrm{R}=\mathrm{H}$

11; $\mathrm{R}=\mathrm{CH}_{2} \mathrm{CH}_{2} \mathrm{CN}$<smiles>Cc1cccc2c1C(CCC#N)(CCC#N)C=CC2=O</smiles><smiles>COc1cccc2c1C(CCC#N)(CCC#N)CC([Ge](C)(O)Cl)NC2=O</smiles>

17<smiles>COc1cccc2c1C(CCC#N)(CCC#N)C(O)CC2</smiles>

12<smiles>COc1cccc2c1C(CCC#N)(CCC#N)CCC2=O</smiles>

$15 \mathrm{O}$<smiles>COC(=O)CCC1(CCC(C)=O)CCNC(=O)c2cccc(OC)c21</smiles>

18<smiles>COc1cccc2c1C(CCC#N)(CCC#N)CCC2=N</smiles>

\section{Scheme 2}


Cyanoethylation of tetralone $7 \mathbf{a}$ at $\mathrm{C}-1$ was carried out by reaction with acrylonitrile in THF and $t$ - $\mathrm{BuOH}$ solution in $96 \%$ yield using potassium tert-butoxide as catalyst. The 1,3tetra(cyanoethyl) derivative of $\mathbf{1 1}$ was also isolated in small amounts as a by-product. Ketone $\mathbf{1 0}$ was reduced (96\%) and the corresponding alcohol 12 was quantitatively dehydrated by phosphorus oxychloride in refluxing pyridine. Oxidation of olefin 13 was achieved with chromium(VI) oxide and resulted in ketone 14 in $65 \%$ yield. Ketone 14 was catalytically hydrogenated in quantitative yield and compound 15 was converted to oxime 16 by a conventional method (91\%). The Beckmann rearrangement of oxime 16 was accomplished in dioxane solution at $70{ }^{\circ} \mathrm{C}$ with thionyl chloride, giving the corresponding benzo[ $c$ ]azepinone 17 in 52\% yield. A Pinner reaction was used to convert the nitrile groups into ester substituents; 17 was treated with dry hydrogen chloride gas in refluxing methanol solution and product $\mathbf{1 8}$ was isolated in $96 \%$ yield. The Dieckmann condensation of diester 18 was carried out in benzene in the presence of potassium tert-butoxide forming the desired spiro structure 19 (Scheme 3 ).

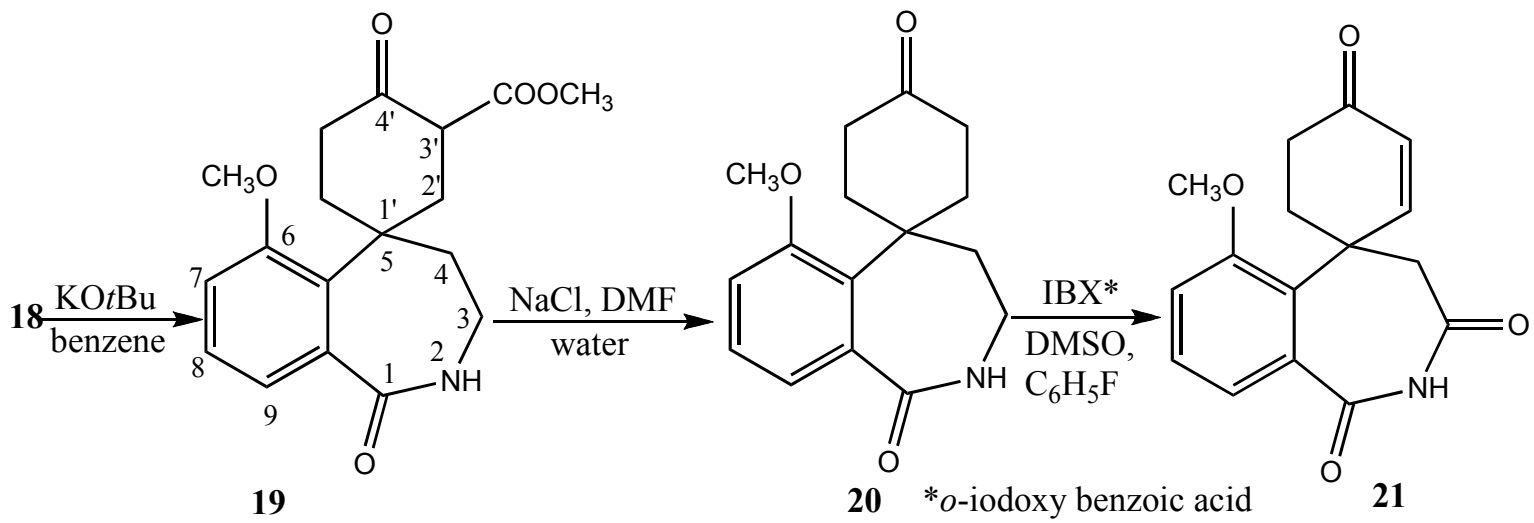

\section{Scheme 3}

Demethoxycarbonylation of 19 furnished spiro ketone $20(62 \%)$, followed by formation of the carbon-carbon double bond in the cyclohexanone ring by the method of Nicolaou et al. ${ }^{13}$ using IBX (o-iodoxy benzoic acid) ${ }^{12}$ as oxidizing agent, giving the unsaturated key intermediate 21 in $62 \%$ yield.

Various reagent systems (boron tribromide, aluminium trichloride/diethyl sulfide, or thiocarbamide, boron trifluoride etherate) were investigated for the $O$-demethylation of imide 21 to initiate the intramolecular addition reaction forming the narwedine-like tetracyclic skeleton. The aluminium trichloride catalyzed reaction with diethyl sulfide in dichloromethane solution resulted in an unexpected product (Scheme 4) as a mixture of two diastereomers (22; 14\%, $\mathrm{D} 1: \mathrm{D} 2 \approx 3: 2)$; in reactions with the other reagents only decomposition products could be obtained. Formation of this type of ethylthio derivative in the demethylation reaction conditions, or similar examples, is unknown in the literature. However, using the methanesulfonic acid/D/Lmethionine demethylating system, the tetracyclic ketone $\mathbf{2 3}$ was obtained almost quantitatively. Although the crude product isolated from the reaction mixture $(93 \%)$ possessed satisfactory 
quality for further reactions, the pure compound was obtained after chromatography in $23.5 \%$ yield.<smiles>COc1cccc2c1C1(C=CC(=O)CC1)CC(=O)NC2=O</smiles>

21<smiles>CC[C@H]1CCC23CC(=O)NC(=O)c4cccc(c42)OC3C1</smiles>

22

D,L-methionine

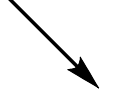

23

\section{Scheme 4}

\section{Conclusions}

The successful preparation of the benzofurobenzazepine tetracycle $\mathbf{2 3}$ confirmed our synthetic strategy to build useful intermediates for preparing compounds like demethoxynarwedine or demethoxylycoraminone.

\section{Experimental Section}

General Procedures. Melting points are uncorrected. IR spectra were recorded on Zeiss IR 75 and 80 instruments. NMR spectra were recorded on a Varian INOVA 300, Varian INOVA 500 
or a Varian VNMRS-500 spectrometer. Chemical shifts are given on the delta scale as parts per million (ppm) with tetramethylsilane (TMS) as the internal standard $(0.00 \mathrm{ppm})$. Mass spectrometric measurements were performed on a VG-Trio-2 and a Finnigan MAT 95SQ mass spectrometer using EI $\left(70 \mathrm{eV}, 220{ }^{\circ} \mathrm{C}\right.$ source temperature) and FIB (Cs ${ }^{+}, 3$-nitrobenzyl alcohol matrix, $20 \mathrm{kV}$ ) ionization methods. High-resolution MS measurements were carried out on a Thermo LTQ FT Ultra mass spectrometer (ESI, 3.5-4.0 kV spray voltage, 275-300 ${ }^{\circ} \mathrm{C}$ capillary temperature, solvent: $\mathrm{MeOH}: \mathrm{H}_{2} \mathrm{O} 1: 1+1 \mathrm{~V} / \mathrm{V} \% \mathrm{cc}$. AcOH). The protonated molecular ion peaks were fragmented by CID at a normalized collision energy of $35 \%$. The relative abundance values of the fragment ions in the MS-MS spectrum are given in brackets. TLC was carried out using Kieselgel $60 \mathrm{~F}_{254}$ (Merck) glass plates. 1,7-Dihydroxynaphthalene (8) was purchased from Aldrich. 1,7-Dimethoxynaphthalene ${ }^{10,11}$ (9), 8-methoxy-2-tetralone ${ }^{11}$ (7a) and IBX $^{12}$ were prepared by literature methods.

3,3'-(8-Methoxy-2-oxo-3,4-dihydronaphthalene-1,1(2H)-diyl)di(propanenitrile) (10). To a stirred solution of 8-methoxy-2-tetralone (7a) $(4.0 \mathrm{~g}, 22.7 \mathrm{mmol})$ in tert-butanol (22 $\mathrm{mL})$, acrylonitrile $(2.7 \mathrm{~mL}, 2.13 \mathrm{~g}, 40.2 \mathrm{mmol})$ was added in THF (5.4 mL) under Ar. Then potassium tert-butoxide (550 mg, $4.9 \mathrm{mmol})$ was added is small portions $(20-30 \mathrm{mg})$ over $10 \mathrm{~min}$ until the reaction temperature increased to $40-50{ }^{\circ} \mathrm{C}$. The reaction mixture was then stirred for $20 \mathrm{~min}$, poured into water $(130 \mathrm{~mL})$ and extracted with dichloromethane $(3 \times 130 \mathrm{~mL})$. The combined organic layers were washed with water, dried $\left(\mathrm{MgSO}_{4}\right)$ and evaporated to dryness under reduced pressure. The residue was purified by column chromatography on silica gel (dichloromethanemethanol 50:1) to yield dinitrile 10 (6.1 g (96\%), mp 109-110 ${ }^{\circ} \mathrm{C}$. TLC $\left(\mathrm{CH}_{2} \mathrm{Cl}_{2}-\mathrm{MeOH} 50: 1\right) R_{f}$ 0.6; IR (KBr): 2220, 1705, 1575, 1460, 1250, 1050, 775, $750 \mathrm{~cm}^{-1} .{ }^{1} \mathrm{H}-\mathrm{NMR}\left(300 \mathrm{MHz}, \mathrm{CDCl}_{3}\right)$ : $\delta 1.88-2.10(\mathrm{~m}, 4 \mathrm{H}), 2.43-2.63\left(\mathrm{~m}, 4 \mathrm{H}, \mathrm{CH}_{2}\right), 2.71\left(\mathrm{t}, 2 \mathrm{H}, \mathrm{CH}_{2}\right), 3.07\left(\mathrm{t}, 2 \mathrm{H}, \mathrm{CH}_{2}\right), 3.89(\mathrm{~s}, 3 \mathrm{H}$, $\left.\mathrm{OCH}_{3}\right), 6.80-6.85(\mathrm{~m}, 2 \mathrm{H}, 2 \times \mathrm{ArH}), 7.28(\mathrm{t}, 1 \mathrm{H}, \mathrm{ArH})$. HRMS: $\mathrm{M}+\mathrm{H}: m / z$ 283.14451, calculated value for $\mathrm{C}_{17} \mathrm{H}_{19} \mathrm{~N}_{2} \mathrm{O}_{2}$ : 283.14410 (delta: 1.43 ppm). MS-MS of $m / z 283$ (\%): $m / z 266$ (61), 255 (100), 249 (5), 242 (4), 238 (22), 229 (24), 224 (12), 200 (4), 173 (12).

A side product was also isolated $(0.18 \mathrm{~g}, 2 \%)$ and was identified as the corresponding 1,3tetracyanoethyl derivative (11); mp $132-133^{\circ} \mathrm{C}$ (dichloromethane-ethanol). TLC $\left(\mathrm{CH}_{2} \mathrm{Cl}_{2}-\mathrm{MeOH}\right.$ 50:1) $R_{f}$ 0.3; IR (KBr): 2230, 1700, 1580, 1480, 1270, $770 \mathrm{~cm}^{-1} .{ }^{1} \mathrm{H}-\mathrm{NMR}\left(300 \mathrm{MHz}, \mathrm{CDCl}_{3}\right): \delta$ 1.80-2.41 (m, 14H), 2.62-2.74 (m, 2H, CH$), 3.00$ (s, 2H, $\left.\mathrm{CH}_{2}\right), 3.91$ (s, 3H, $\left.\mathrm{OCH}_{3}\right), 6.85$ (dd, 1H, ArH), 6.95 (dd, 1H, ArH), 7.36 (d, 1H, ArH). MS (EI) m/z (\%): 388(M $\left.\mathrm{M}^{+}, 30\right), 348(100)$, 334(66), 281(19), 254(21), 240(29), 212(23), 159(22), 115(30). Anal. Calcd for $\mathrm{C}_{23} \mathrm{H}_{24} \mathrm{~N}_{4} \mathrm{O}_{2}$ : C 71.11, H 6.23, N 14.42. Found C 70.94, H 6.22, N 14.36\%.

\section{3,3'-(2-Hydroxy-8-methoxy-3,4-dihydronaphthalene-1,1(2H)-diyl)di(propanenitrile) (12).}

Ketone 10 (3.22 g, $11.4 \mathrm{mmol})$ was dissolved in a mixture of $\mathrm{MeOH}(222 \mathrm{~mL})$ and water (3 $\mathrm{mL})$ and sodium borohydride $(1.2 \mathrm{~g}, 34.4 \mathrm{mmol})$ was added in small portions over $1.5 \mathrm{~h}$ at $0{ }^{\circ} \mathrm{C}$ with stirring. After stirring at $0{ }^{\circ} \mathrm{C}$ for $20 \mathrm{~min}$ the reaction mixture was acidified ( $\mathrm{pH} 5$ ) with acetic acid. The solvent was evaporated under reduced pressure, 10\% aqueous sodium carbonate (50 $\mathrm{mL})$ and water $(50 \mathrm{~mL})$ was added to the residue and the mixture was extracted with 
dichloromethane $(3 \times 100 \mathrm{~mL})$. The combined organic layers were washed with water $(2 \times 150$ $\mathrm{mL})$, dried $\left(\mathrm{MgSO}_{4}\right)$ and evaporated to dryness under reduced pressure to yield pure alcohol 12 (3.01 g, 96\%), mp $82{ }^{\circ} \mathrm{C}$. TLC $\left(\mathrm{CH}_{2} \mathrm{Cl}_{2}-\mathrm{MeOH} 50: 1\right) R_{f} 0.43$; IR(KBr) 3500, 2230, 1470, 1460, $1205 \mathrm{~cm}^{-1} .{ }^{1} \mathrm{H}$ NMR $\left(300 \mathrm{MHz}, \mathrm{CDCl}_{3}\right) \delta 1.83-2.60\left(\mathrm{~m}, 10 \mathrm{H}, \mathrm{CH}_{2}\right), 2.75-2.95\left(\mathrm{~m}, 2 \mathrm{H}, \mathrm{CH}_{2}\right)$, $3.86\left(\mathrm{~s}, 3 \mathrm{H}, \mathrm{OCH}_{3}\right), 3.88-3.94(\mathrm{~m}, 1 \mathrm{H}, \mathrm{OH}), 6.73(\mathrm{~m}, 2 \mathrm{H}, 2 \times \mathrm{ArH}), 7.18(\mathrm{t}, 1 \mathrm{H}, \mathrm{ArH}) . \mathrm{MS}(\mathrm{EI})$ m/z (\%): 284 ( $\left.\mathrm{M}^{+}, 100\right), 267$ (39), 230 (27), 226 (32), 212 (36), 203 (44), 185 (21), 171 (32), 159 (24), 147 (18), 131 (44), 115 (85). Anal. Calcd for $\mathrm{C}_{17} \mathrm{H}_{20} \mathrm{~N}_{2} \mathrm{O}_{2}$ : C 71.81, H 7.09, N 9.85. Found C 71.58, H 7.14, N 9.76\%.

3,3'-(8-Methoxynaphthalene-1,1(4H)-diyl)di(propanenitrile) (13). To a solution of hydroxy derivative $12(2.0 \mathrm{~g}, 7.05 \mathrm{mmol})$ in dry pyridine $(14 \mathrm{~mL})$, phosphorus oxychloride $(0.96 \mathrm{~mL}$, $1.56 \mathrm{~g}, 10.17 \mathrm{mmol}$ ) was added with stirring. After refluxing for $3 \mathrm{~h}$ at $160{ }^{\circ} \mathrm{C}$ the reaction mixture was evaporated to dryness under reduced pressure, the residue was poured into ice and acidified with a mixture of water and conc. hydrochloric acid 1:1 to $\mathrm{pH} 1$. The acidic mixture was then washed with dichloromethane $(3 \times 20 \mathrm{~mL})$, the combined organic layers after drying $\left(\mathrm{MgSO}_{4}\right)$ were evaporated to dryness leaving oily product (13) (1.87 g, 99\%) which spontaneously crystallized in a refrigerator, mp 108-110 ${ }^{\circ} \mathrm{C}$. TLC $\left(\mathrm{CH}_{2} \mathrm{Cl}_{2}-\mathrm{MeOH}\right.$ 50:1) $R_{f} 0.8$; IR (KBr) 2225, 1600, 1580, 1460, 1260, 1070, 790, $720 \mathrm{~cm}^{-1} .{ }^{1} \mathrm{H}$ NMR $\left(300 \mathrm{MHz}, \mathrm{CDCl}_{3}\right) \delta$ 1.61-1.66 (m, 2H), 1.74-1.82 (m, 2H), 2.08-2.15 (m, 2H), 2.84-2.92 (m, 2H), $3.40(\mathrm{~m}, 2 \mathrm{H}), 3.87$ $\left(\mathrm{s}, 3 \mathrm{H}, \mathrm{OCH}_{3}\right), 5.24(\mathrm{dt}, 1 \mathrm{H}, \mathrm{C}-\underline{\mathrm{CH}}=\mathrm{CH}), 6.15\left(\mathrm{dt}, 1 \mathrm{H}, \mathrm{CH}=\underline{\mathrm{CH}}-\mathrm{CH}_{2}\right), 6.75-6.78(\mathrm{~m}, 2 \mathrm{H}, 2 \times$ ArH), 7.22 (t, 1H, ArH). MS (EI) m/z (\%): $266\left(\mathrm{M}^{+}, 21\right), 250$ (6), 226 (10), 212 (84), 197 (7), 171 (100), 141 (20), 128 (17), 115 (26). Anal. Calcd for $\mathrm{C}_{17} \mathrm{H}_{18} \mathrm{~N}_{2} \mathrm{O}$ : C 76.66, H 6.81, N 10.52. Found C 76.38, H 6.81, N 10.38\%.

3,3'-(8-Methoxy-4-oxonaphthalene-1,1(4H)-diyl)di(propanenitrile) (14). Unsaturated derivative 13 (2.6 g, $9.7 \mathrm{mmol})$ was dissolved in acetic acid $(100 \mathrm{~mL})$ and $\mathrm{CrO}_{3}(3.6 \mathrm{~g}, 35.8$ mmol) was added dropwise in acetic acid $(50 \mathrm{~mL})$-water $(5 \mathrm{~mL})$ solution at $14-16{ }^{\circ} \mathrm{C}$ over $1 \mathrm{~h}$ under stirring. The reaction mixture was stirred at room temperature for $1 \mathrm{~h}$ and isopropanol (100 $\mathrm{mL}$ ) was added dropwise under external ice-water cooling. After stirring for $1 \mathrm{~h}$ at room temperature the reaction mixture was evaporated to dryness in vacuum. The residue was suspended in water $(160 \mathrm{~mL})$ and extracted with dichloromethane $(3 \times 160 \mathrm{~mL})$. The combined organic layers were washed with $10 \%$ aqueous sodium hydrogencarbonate $(5 \times 100 \mathrm{~mL})$ and with brine $(100 \mathrm{~mL})$. After drying $\left(\mathrm{MgSO}_{4}\right)$, the solvent was evaporated to dryness under reduced pressure and the residue was purified by column chromatography on silica gel (dichloromethane-methanol 50:1) yielding of unsaturated ketone 14 (2.35 g, 65\%), mp 142-143 ${ }^{\circ} \mathrm{C}$ (petroleum ether [40-70]-THF). TLC $\left(\mathrm{CH}_{2} \mathrm{Cl}_{2}-\mathrm{MeOH} 50: 1\right) R_{f} 0.27$; IR (KBr) 2220, 1680, 1650, 1600, 1580, 1320, 1270, 1060, 810, $770 \mathrm{~cm}^{-1} .{ }^{1} \mathrm{H}$ NMR $\left(300 \mathrm{MHz}, \mathrm{CDCl}_{3}\right) \delta 1.66-1.78(\mathrm{~m}$, $2 \mathrm{H}), 1.90-2.05(\mathrm{~m}, 4 \mathrm{H}), 3.15-3.22(\mathrm{~m}, 2 \mathrm{H}), 3.99\left(\mathrm{~s}, 3 \mathrm{H}, \mathrm{OCH}_{3}\right), 6.61(\mathrm{~d}, 1 \mathrm{H}, \mathrm{CH}=\mathrm{CH}), 6.69(\mathrm{~d}$, $1 \mathrm{H}, \mathrm{CH}=\mathrm{CH}), 7.20(\mathrm{dd}, 1 \mathrm{H}, \mathrm{ArH}), 7.53$ (dd, 1H, ArH), 7.91 (dd, 1H, ArH). HRMS: M+H: $m / z$ 281.12837, calculated value for $\mathrm{C}_{17} \mathrm{H}_{17} \mathrm{~N}_{2} \mathrm{O}_{2}$ : 281.12845 (delta: $-0.30 \mathrm{ppm}$ ). MS-MS of $\mathrm{m} / z$ 281: $m / z 264$ (4), 250 (2), 240 (100), 227 (25). 
3,3'-(8-Methoxy-4-oxo-3,4-dihydronaphthalene-1,1(2H)-diyl)di(propanenitrile)

(15).

Unsaturated ketone 14 (2.05 g, $7.33 \mathrm{mmol})$ was hydrogenated in a mixture of dichloromethane $(93 \mathrm{~mL})$ and ethanol $(93 \mathrm{~mL})$ in the presence of $10 \%$ palladium on charcoal $(0.4 \mathrm{~g})$ at room temperature under atmospheric pressure. The catalyst was filtered off, the filtrate was evaporated to dryness to give the saturated product $15(2.07 \mathrm{~g}, 100 \%), \mathrm{mp} 105-107{ }^{\circ} \mathrm{C}$. TLC (toluene-MeOH 7:1) $R_{f}$ 0.42; IR (KBr) 2225, 1695, 1460, $1270 \mathrm{~cm}^{-1} .{ }^{1} \mathrm{H}$ NMR $\left(300 \mathrm{MHz}, \mathrm{CDCl}_{3}\right) \delta 2.02-2.30(\mathrm{~m}$, $8 \mathrm{H}), 2.58-2.70(\mathrm{~m}, 4 \mathrm{H}), 3.93\left(\mathrm{~s}, 3 \mathrm{H}, \mathrm{OCH}_{3}\right), 7.16(\mathrm{dd}, 1 \mathrm{H}, \mathrm{ArH}), 7.40(\mathrm{t}, 1 \mathrm{H}, \mathrm{ArH}), 7.76(\mathrm{dd}, 1 \mathrm{H}$, ArH). MS (FIB) m/z: $283\left(\mathrm{MH}^{+}\right)$; MS-MS of $m / z$ 283: 266 (100), 248 (77), 228 (53), 176 (35), 161 (20), 135 (11). Anal. Calcd for $\mathrm{C}_{17} \mathrm{H}_{18} \mathrm{~N}_{2} \mathrm{O}_{2}$ : C 72.32, H 6.42, N 9.92. Found $\mathrm{C} 72.30, \mathrm{H}$ $6.40, \mathrm{~N} 9.77 \%$.

\section{3,3'-(4-Hydroxyimino-8-methoxy-3,4-dihydronaphthalene-1,1(2H)-diyl)di(propanenitrile)}

(16). To a solution of ketone $15(1.33 \mathrm{~g}, 4.7 \mathrm{mmol})$ in ethanol (34 $\mathrm{mL})$ was added hydroxylamine hydrochloride $(0.5 \mathrm{~g}, 7.1 \mathrm{mmol})$ dissolved in water $(34 \mathrm{~mL})$ and then sodium acetate $(0.39 \mathrm{~g}$, $4.74 \mathrm{mmol}$ ) was added. The reaction mixture was refluxed for $3 \mathrm{~h}$ and then it was poured into water $(30 \mathrm{~mL})$. The mixture was extracted with dichloromethane $(3 \times 20 \mathrm{~mL})$, the combined organic layers were dried $\left(\mathrm{MgSO}_{4}\right)$ and concentrated under reduced pressure. The oily residue crystallized by treatment of a few drops of hexane to give product (16) $(1.27 \mathrm{~g}, 91 \%), \mathrm{mp} 120$ $121{ }^{\circ} \mathrm{C}$. TLC $\left(\mathrm{CH}_{2} \mathrm{Cl}_{2}-\mathrm{MeOH} 40: 1\right) R_{f} 0.4$; IR (KBr) 3500, 2225, 1310, 1270, $960 \mathrm{~cm}^{-1}$. ${ }^{1} \mathrm{H}$ NMR $\left(300 \mathrm{MHz}, \mathrm{CDCl}_{3}\right) \delta 1.78-1.82(\mathrm{~m}, 2 \mathrm{H}), 1.86-2.02(\mathrm{~m}, 2 \mathrm{H}), 2.16-2.24(\mathrm{~m}, 4 \mathrm{H}), 2.50-2.60(\mathrm{~m}$, 2H) 2.80-2.86 (m, 2H), $3.89\left(\mathrm{~s}, 3 \mathrm{H}, \mathrm{OCH}_{3}\right), 6.94(\mathrm{br}, 1 \mathrm{H}, \mathrm{N}-\mathrm{OH}), 6.94(\mathrm{dd}, 1 \mathrm{H}, \mathrm{ArH}), 7.26(\mathrm{t}$, 1H, ArH), 7.64 (dd, 1H, ArH). MS (EI) m/z (\%): $297\left(\mathrm{M}^{+}, 100\right), 282$ (14), 243 (66), 225 (60), 210 (34), 198 (34), 183 (41), 171 (43), 156 (49), 146 (27), 128 (27), 115 (35). Anal. Calcd for $\mathrm{C}_{17} \mathrm{H}_{19} \mathrm{~N}_{3} \mathrm{O}_{2}$ : C 68.67, H 6.44, N 14.13. Found C 68.52, H 6.46, N 13.99\%.

3,3'-(6-Methoxy-1-oxo-1,2,3,4-tetrahydro-2-benzazepin-5,5-diyl)di(propanenitrile)

(17).

Oxime 16 (7.3 g, $24.5 \mathrm{mmol})$ was dissolved in dioxane $(134 \mathrm{~mL})$ and thionyl chloride $(9.2 \mathrm{~mL}$, $15 \mathrm{~g}, 126 \mathrm{mmol})$ was added dropwise in dioxane solution $(70 \mathrm{~mL})$ at $70{ }^{\circ} \mathrm{C}$ with stirring. Then the reaction mixture was stirred at $70{ }^{\circ} \mathrm{C}$ for a further $2 \mathrm{~h}$, poured into a mixture of saturated aqueous sodium carbonate $(400 \mathrm{~mL})$ and ice $(\mathrm{pH}$ must be maintained 9-10) and was extracted with dichloromethane $(3 \times 150 \mathrm{~mL})$. The organic layers were combined, after drying $\left(\mathrm{MgSO}_{4}\right)$ the solvent was evaporated under reduced pressure and the residue was purified by column chromatography on silica gel (dichloromethane-methanol 40:1) to yield product (17) (3.8 g, 52\%), mp 170-172 ${ }^{\circ} \mathrm{C}$. TLC $\left(\mathrm{CH}_{2} \mathrm{Cl}_{2}-\mathrm{MeOH} 20: 1\right) R_{f}$ 0.38; IR (KBr) 3400, 2220, 1650, 1570, 1450, 1250, 1045, 900, $750 \mathrm{~cm}^{-1} .{ }^{1} \mathrm{H}$ NMR (300 MHz, $\left.\mathrm{CDCl}_{3}\right) \delta 1.96-2.02(\mathrm{~m}, 2 \mathrm{H}), 2.08-2.24$ (m, 6H), 2.38-2.48 (m, 2H), $3.17\left(\mathrm{~m}, 2 \mathrm{H}, \mathrm{CH}_{2} \mathrm{~N}\right), 3.84\left(\mathrm{~s}, 3 \mathrm{H}, \mathrm{OCH}_{3}\right), 7.02(\mathrm{dd}, 1 \mathrm{H}, \mathrm{ArH}), 7.32$ (brt, 1H, NH), 7.32 (t, 1H, ArH), 7.42 (dd, 1H, ArH). HRMS: M+H: $m / z$ 298.15506, calculated value for $\mathrm{C}_{17} \mathrm{H}_{20} \mathrm{~N}_{3} \mathrm{O}_{2}$ : 298.15500 (delta: $0.19 \mathrm{ppm}$ ). MS-MS of $\mathrm{m} / z$ 298: $\mathrm{m} / z 280$ (100), 269 (28), 263 (19), 226 (13), 212 (6), 200 (4).

Dimethyl 3,3'-(6-methoxy-1-oxo-1,2,3,4-tetrahydro-2-benzazepin-5,5-diyl)-di(propanoate) (18). Into a suspension of dinitrile $17(3.84 \mathrm{~g}, 12.9 \mathrm{mmol})$ in methanol $(60 \mathrm{~mL})$, dry $\mathrm{HCl}$ gas was introduced for $1 \mathrm{~h}$. Then the reaction mixture was refluxed for $1 \mathrm{~h}$, and then it was poured into a 
mixture of ice and saturated aqueous sodium carbonate solution $(350 \mathrm{~mL})$ and extracted with dichloromethane $(3 \times 130 \mathrm{~mL})$. The combined organic layers were dried $\left(\mathrm{MgSO}_{4}\right)$, the solvent was evaporated to dryness under reduced pressure and the residue was treated with hexane to give of diester 18 (4.5 g, 96\%), mp $121{ }^{\circ} \mathrm{C}$. TLC $\left(\mathrm{CH}_{2} \mathrm{Cl}_{2}-\mathrm{MeOH} 20: 1\right) R_{f} 0.43$; IR (KBr) 1740, $1665,1570,1200,1180,990,800 \mathrm{~cm}^{-1} .{ }^{1} \mathrm{H}$ NMR $\left(300 \mathrm{MHz}, \mathrm{CDCl}_{3}\right) \delta 2.02-2.08(\mathrm{~m}, 2 \mathrm{H}), 2.10-$ $2.25(\mathrm{~m}, 6 \mathrm{H}), 2.39-2.49(\mathrm{~m}, 2 \mathrm{H}), 3.23\left(\mathrm{~m}, 2 \mathrm{H}, \mathrm{CH}_{2} \mathrm{~N}\right), 3.62\left(\mathrm{~s}, 6 \mathrm{H}, 2 \times \mathrm{COOCH}_{3}\right), 3.83(\mathrm{~s}, 3 \mathrm{H}$, $\left.\mathrm{OCH}_{3}\right), 6.86$ (brm, 1H, NH), 7.03 (dd, 1H, ArH), 7.30 (t, 1H, ArH), 7.50 (dd, 1H, ArH). HRMS: $\mathrm{M}+\mathrm{H}: \mathrm{m} / z$ 364.17551, calculated value for $\mathrm{C}_{19} \mathrm{H}_{26} \mathrm{NO}_{6}: 364.17546$ (delta: $0.13 \mathrm{ppm}$ ). MS-MS of $\mathrm{m} / \mathrm{z}$ 364: $\mathrm{m} / \mathrm{z} 332$ (100), 314 (6).

Methyl 1,4'-dioxo-6-methoxy-1,2,3,4-tetrahydrospiro[2-benzazepine-5,1'-cyclohexane]-3'carboxylate (19). To a solution of diester $18(5.66 \mathrm{~g}, 15.6 \mathrm{mmol})$ in dry benzene $(220 \mathrm{~mL})$, potassium tert-butoxide $(3.96 \mathrm{~g}, 35.3 \mathrm{mmol})$ was added and the reaction mixture was refluxed for $30 \mathrm{~min}$. After cooling to room temperature the mixture was evaporated to dryness, water (100 $\mathrm{mL}$ ) was added and the solution was acidified with $2 \mathrm{~N}$ hydrochloric acid to $\mathrm{pH} 2(22 \mathrm{~mL})$. After extracting with dichloromethane $(3 \times 100 \mathrm{~mL})$, the combined organic layers were dried $\left(\mathrm{MgSO}_{4}\right)$ and the solvent was evaporated to yield $\beta$-oxoester derivative $19(4.4 \mathrm{~g}, 85 \%)$ as a foam. TLC $\left(\mathrm{CH}_{2} \mathrm{Cl}_{2}-\mathrm{MeOH} 20: 1\right) R_{f}$ 0.5; IR (KBr) 1660, 1620, 1450, 1210, $1050 \mathrm{~cm}^{-1} .{ }^{1} \mathrm{H}$ NMR (300 MHz, $\mathrm{CDCl}_{3}$ ) (in enolic form) $\delta 1.60-2.20(\mathrm{~m}, 6 \mathrm{H}), 2.60-2.70(\mathrm{~m}, 1 \mathrm{H}), 3.10-3.22(\mathrm{~m}, 2 \mathrm{H}), 3.50-3.62(\mathrm{~d}$, $1 \mathrm{H}), 3.63\left(\mathrm{~s}, 3 \mathrm{H}, \mathrm{COOCH}_{3}\right), 3.82\left(\mathrm{~s}, 3 \mathrm{H}, \mathrm{OCH}_{3}\right), 6.70$ (brm, 1H, NH), 6.98 (dd, 1H, ArH), 7.28 (t, 1H, ArH), 7.35 (dd, 1H, ArH), 11.93 (s, 1H, OH). HRMS: M+H: $m / z$ 332.14937, calculated value for $\mathrm{C}_{18} \mathrm{H}_{22} \mathrm{NO}_{5}$ : 332.14925 (delta: $0.36 \mathrm{ppm}$ ). MS-MS of $\mathrm{m} / \mathrm{z} 332: \mathrm{m} / \mathrm{z} 300$.

6-Methoxy-3,4-dihydrospiro[2-benzazepine-5,1'-cyclohexane]-1(2H), $\mathbf{4}^{\prime}$-dione (20). To a solution of ketoester $19(1.46 \mathrm{~g}, 4.4 \mathrm{mmol})$ in DMF $(22 \mathrm{~mL})$, water $(0.15 \mathrm{~mL})$ and sodium chloride $(0.26 \mathrm{~g}, 4.4 \mathrm{mmol})$ were added. The reaction mixture was stirred at $150{ }^{\circ} \mathrm{C}$ for $3 \mathrm{~h}$ under argon, and then it was evaporated to dryness under vacuum. The residue was partitioned between dichloromethane $(80 \mathrm{~mL})$ and brine $(50 \mathrm{~mL})$, the organic phase was washed with dichloromethane $(3 \times 70 \mathrm{~mL})$ and the combined organic layers were dried $\left(\mathrm{MgSO}_{4}\right)$ and evaporated to dryness under reduced pressure. The residue was purified by preparative thin layer chromatography on silica gel $\left(\mathrm{CH}_{2} \mathrm{Cl}_{2}-\mathrm{MeOH} 20: 1\right)$ to yield $20(0.78 \mathrm{~g}, 65 \%), \mathrm{mp} 213{ }^{\circ} \mathrm{C}$. TLC $\left(\mathrm{CH}_{2} \mathrm{Cl}_{2}-\mathrm{MeOH} 20: 1\right) R_{f}$ 0.4; IR (KBr) 3320, 1710, 1650, 1620, 1450, 1250, $760 \mathrm{~cm}^{-1}$. ${ }^{1} \mathrm{H}-\mathrm{NMR}$ $\left(300 \mathrm{MHz}, \mathrm{CDCl}_{3}\right) \delta 1.70-1.81(\mathrm{~m}, 2 \mathrm{H}), 1.94-2.02(\mathrm{~m}, 2 \mathrm{H}), 2.20-2.25(\mathrm{~m}, 4 \mathrm{H}), 2.86-3.00(\mathrm{~m}$, 2H) $3.04-3.10(\mathrm{~m}, 2 \mathrm{H}), 3.76\left(\mathrm{~s}, 3 \mathrm{H}, \mathrm{OCH}_{3}\right), 6.73$ (brm, 1H, NH), 7.01 (dd, 1H, ArH), $7.28(\mathrm{t}, 1 \mathrm{H}$, ArH), 7.33 (dd, 1H, ArH). HRMS: M+H: $m / z$ 274.14381, calculated value for $\mathrm{C}_{16} \mathrm{H}_{20} \mathrm{NO}_{3}$ : 274.14377 (delta: 0.14 ppm). MS-MS of $m / z$ 274: $m / z 256$ (100), 242 (30), 239 (18), 227 (47), 213 (20), 203 (26), 187 (20), 185 (12), 166 (11), 152 (9), 135 (45).

6-Methoxyspiro[2-benzazepine-5,1'-cyclohexan]-2'-ene-1,3(2H,4H),4'-trione (21). Lactam (20) $(2.87 \mathrm{~g}, 10.5 \mathrm{mmol})$ was dissolved in a mixture of fluorobenzene $(74 \mathrm{~mL})$ and DMSO (37 $\mathrm{mL})$ and $o$-iodoxybenzoic acid $(11.46 \mathrm{~g}, 40.9 \mathrm{mmol})^{12}$ was added. The reaction mixture was heated at $85{ }^{\circ} \mathrm{C}$ with stirring under argon for $77 \mathrm{~h}$. Then the solvent was evaporated under vacuum, the residue was taken up in dichloromethane $(500 \mathrm{~mL})$, washed with saturated aqueous 
sodium hydrogen carbonate $(2 \times 150 \mathrm{~mL})$, with water $(250 \mathrm{~mL})$ and with saturated aqueous sodium chloride solution $(2 \times 150 \mathrm{~mL})$. The organic layer was dried $\left(\mathrm{MgSO}_{4}\right)$ and evaporated to dryness under reduced pressure and the residue was treated with dichloromethane to yield $1.23 \mathrm{~g}$ of product. A further $377 \mathrm{mg}$ of $\mathbf{2 1}$ was isolated from the mother liquor of dichloromethane by preparative thin layer chromatography on silica gel $\left(\mathrm{CH}_{2} \mathrm{Cl}_{2}-\mathrm{MeOH} 20: 1\right)$ giving a total yield of 21 (1.61 g, 54\%), mp 131-134 ${ }^{\circ} \mathrm{C}$. TLC $\left(\mathrm{CH}_{2} \mathrm{Cl}_{2}-\mathrm{MeOH} 20: 1\right) R_{f}$ 0.50; IR (KBr) 1705, 1665, $1650,1630,1300,810,740 \mathrm{~cm}^{-1} .{ }^{1} \mathrm{H}$ NMR (300 MHz, DMSO-d 6 ) $\delta 2.00-2.08(\mathrm{~m}, 1 \mathrm{H}), 2.30-2.76$ $(\mathrm{m}, 4 \mathrm{H}), 3.52(\mathrm{~d}, 1 \mathrm{H}), 3.76\left(\mathrm{~s}, 3 \mathrm{H}, \mathrm{OCH}_{3}\right), 5.80(\mathrm{~d}, 1 \mathrm{H}, \mathrm{CH}), 7.00(\mathrm{~d}, 1 \mathrm{H}, \mathrm{CH}), 7.37(\mathrm{dd}, 1 \mathrm{H}$, ArH), 7.47 (t, 1H, ArH), 7.95 (dd, 1H, ArH), 11.15 (brs, 1H, NH). HRMS: M+H: m/z 286.10740, calculated value for $\mathrm{C}_{16} \mathrm{H}_{16} \mathrm{NO}_{4}: 286.10738$ (delta: $0.05 \mathrm{ppm}$ ). MS-MS of $\mathrm{m} / z$ 286: $\mathrm{m} / z 268$ (100), 258 (20), 251 (9), 244 (34), 240 (35), 229 (16), 226 (43), 213 (37).

\section{6-(Ethylsulfanyl)-5,6,7,8-tetrahydro-4a $H$-[1]benzofuro[3a,3,2-ef][2]benzazepine-}

10(9H),12(11H)-dione (22). To a mixture of dichloromethane (14 $\mathrm{mL})$ and aluminium trichloride (431 mg, $3.2 \mathrm{mmol})$, diethyl sulfide $(2 \mathrm{~mL}, 1.7 \mathrm{~g}, 19 \mathrm{mmol})$ and 21 (250 mg, 0.88 $\mathrm{mmol})$ in dichloromethane $(7 \mathrm{~mL})$ solution was added with stirring at room temperature. After stirring for $4 \mathrm{~h}$ the reaction mixture was poured into a mixture of water $(15 \mathrm{~mL})$ and methanol $(25 \mathrm{~mL})$ and extracted with dichloromethane $(3 \times 20 \mathrm{~mL})$. The combined organic layers were washed with brine $(3 \times 15 \mathrm{~mL})$, dried $\left(\mathrm{NaSO}_{4}\right)$ and evaporated to dryness under reduced pressure. The residue was purified by preparative thin layer chromatography on silica gel $\left(\mathrm{CH}_{2} \mathrm{Cl}_{2}-\mathrm{MeOH} 10: 1\right)$ to yield tetracycle $22(39 \mathrm{mg}, 14 \%)$ as an oil. TLC $\left(\mathrm{CH}_{2} \mathrm{Cl}_{2}-\mathrm{MeOH} 10: 1\right)$ $R_{f}$ 0.78; IR (KBr) 3450, 1710, 1695(br), 1675, 1610, 1310, 1250, 820, $760 \mathrm{~cm}^{-1} .{ }^{1} \mathrm{H}$ NMR (500 $\left.\mathrm{MHz}, \mathrm{CDCl}_{3}\right) \delta 1.26\left(\mathrm{t}, \mathrm{CH}_{3}-\mathrm{D} 2\right), 1.28\left(\mathrm{t}, \mathrm{CH}_{3}-\mathrm{D} 1\right), 1.41-1.51(\mathrm{~m}, \mathrm{H}-7 \mathrm{ax}-\mathrm{D} 1), 1.56-1.78(\mathrm{~m}, \mathrm{H}-$ 8ax-D1, H-7eq-D2, H-5ax-D1, H-8eq-D2), 1.86-2.00 (m, H-7ax-D2, H-7eq-D1), 2.01-2.08 (m, H-8eq-D1), 2.09-2.15 (m, H-8ax-D2), 2.26 (ddd, H-5ax-D2), 2.46-2.52 (H-5eq-D2), 2.56 (q, $\left.\mathrm{CH}_{2} \mathrm{~S}-\mathrm{D} 2\right), 2.62$ (q, 2H, CH $2 \mathrm{~S}-\mathrm{D} 1$ ), 2.62-2.70 (m, H-5eq-D1), 2.94 (d, $J_{\text {gem }}=15.4 \mathrm{~Hz}, \mathrm{H}-9 \mathrm{~A}-$ D1), 2.96 (d, $J_{\text {gem }}=15.4$ Hz, H-9A-D2), 2.94-3.02 (m, H-6ax-D1), 3.05 (d, $J_{\text {gem }}=15.4$ Hz, H9B-D2), 3.15 (d, $J_{\text {gem }}=15.4$ Hz, H-9B-D1), 3.15-3.22 (m, H-6eq-D2), 4.33-4.36 (m, Heq-4a-D1), 4.36-4.40 (m, Heq-4a-D2), 7.09 (d, H-3-D1), 7.13 (d, H-3-D2), 7.33 (2×t, H-2-D2, H-2-D1), 7.73 (d, H-1-D2), 7.75 (d, H-1-D1), 8.25 (brs, NH). ${ }^{13} \mathrm{C}-\mathrm{NMR}\left(125 \mathrm{MHz}, \mathrm{CDCl}_{3}\right) 14.6\left(\mathrm{CH}_{3}-\mathrm{D} 2\right)$, 15.0 ( $\mathrm{CH}_{3}$-D1), 24.2 ( $\left.\mathrm{CH}_{2} \mathrm{~S}-\mathrm{D} 1\right), 24.9$ (C-7-D2), 26.1 ( $\left.\mathrm{CH}_{2} \mathrm{~S}-\mathrm{D} 2\right), 26.8$ (C-8-D2), 27.4 (C-7-D1), 30.5 (C-5-D2), 30.8 (C-8-D1), 32.4 (C-5-D1), 36.7 (C-6-D1), 36.9 (C-6-D2), 41.4 (C-8a-D1), 41.9 (C-9-D1), 42.0 (C-8a-D2), 43.6 (C-9-D2), 86.8 (C-4a-D2), 88.2 (C-4a-D1), 115.7 (C-3-D1), 115.8 (C-3-D2), 124.1 (C-1-D2), 124.2 (C-1-D1), 125.1 (C-12a), 129.2 (C-2-D1), 129.3 (C-2D2), 136.1 (C-12b-D2), 137.0 (C-12b-D1), 157.8 (C-3a-D1), 158.0 (C-3a-D2), 163.6 (CO-12D1), 163.7 (CO-12-D2), 169.8 (CO-10-D1), 170.0 (CO-10-D2). HRMS: M+H: $m / z$ 318.11588, calculated value for $\mathrm{C}_{17} \mathrm{H}_{20} \mathrm{NO}_{3} \mathrm{~S}: 318.11584$ (delta: $0.12 \mathrm{ppm}$ ). MS-MS of $\mathrm{m} / z$ 318: $\mathrm{m} / \mathrm{z} 301$ (6), 276 (100), 259 (12), 214 (6).

7,8-Dihydro-4aH-[1]benzofuro[3a,3,2-ef][2]benzazepine-6(5H),10(9H),12(11H)-trione (23). Unsaturated spiroketone (21) $(250 \mathrm{mg}, 0.88 \mathrm{mmol})$ was dissolved in methanesulfonic acid (1.7 $\mathrm{mL})$ and $\mathrm{D}, \mathrm{L}-\mathrm{methionine}(143 \mathrm{mg}, 0.96 \mathrm{mmol})$ was added to the solution under stirring at room 
temperature. After $10 \mathrm{~h}$ the reaction mixture was diluted with water $(10 \mathrm{~mL})$ and extracted with dichloromethane $(5 \times 30 \mathrm{~mL})$. The combined organic layers were dried $\left(\mathrm{MgSO}_{4}\right)$, the solvent was evaporated under reduced pressure to yield tetracyclic compound 23 (221 mg, 93\%), which was pure enough for further reactions. After preparative thin layer chromatography on silica gel (benzene-MeOH 14:3) of 23 (56 mg, 23.5\%) was obtained, mp $240{ }^{\circ} \mathrm{C}$. TLC (benzene-MeOH 14:3) $R_{f}$ 0.5; IR (KBr) 3290, 2920, 1730, 1690, 1605, 1355, 1300, 800, $750 \mathrm{~cm}^{-1} .{ }^{1} \mathrm{H}-\mathrm{NMR}(500$ MHz, DMSO- $\left.d_{6}\right) \delta 1.69-1.79(\mathrm{~m}, 1 \mathrm{H}, \mathrm{H}-7 \mathrm{ax}), 2.00-2.06$ (m, 1H, H-8eq), 2.13-2.22 (m, 1H, H8ax), 2.22-2.28 (m, 1H, H-7eq), $2.72\left(\mathrm{dd}, 1 \mathrm{H}, J_{\mathrm{gem}}=17.1 \mathrm{~Hz}, J=3.5 \mathrm{~Hz}, \mathrm{H}-5 \mathrm{~A}\right), 3.03(\mathrm{~d}, 1 \mathrm{H}$, $\left.J_{\text {gem }}=15.2 \mathrm{~Hz}, \mathrm{H}-9 \mathrm{~A}\right), 3.23\left(\mathrm{dd}, 1 \mathrm{H}, J_{\text {gem }}=17.1 \mathrm{~Hz}, J=2.4 \mathrm{~Hz}, \mathrm{H}-5 \mathrm{~B}\right), 3.34\left(\mathrm{~d}, 1 \mathrm{H}, J_{\text {gem }}=15.2\right.$ Hz, H-9B), 4.88-4.92 (m, 1H, Heq-4a), 7.11 (d, 1H, H-3), 7.39 (t, 1H, H-2), 7.60 (d, 1H, H-1), $10.91(\mathrm{~s}, 1 \mathrm{H}, \mathrm{NH}) .{ }^{13} \mathrm{C}-\mathrm{NMR}\left(125 \mathrm{MHz}, \mathrm{DMSO}-d_{6}\right) \delta 29.2(\mathrm{C}-8), 34.8(\mathrm{C}-7), 40.1$ (C-5), 41.7 (C-8a), 46.3 (C-9), 86.6 (C-4a), 114.0 (C-3), 123.8 (C-1), 127.0 (C-12a), 129.6 (C-2), 133.5 (C12b), 158.1 (C-3a), 163.9 (CO-12), 170.8 (CO-10), 208.1 (CO-6). HRMS: M+H: $m / z$ 272.09183, calculated value for $\mathrm{C}_{15} \mathrm{H}_{14} \mathrm{NO}_{4}: 272.09173$ (delta: $0.35 \mathrm{ppm}$ ). MS-MS of $m / z$ 272: $\mathrm{m} / z 254$ (79), 244 (11), 237 (1), 230 (100), 227 (3), 213 (5), 202 (6).

\section{Acknowledgements}

The authors are grateful to Dr. H. Medzihradszky-Schweiger and her staff for the microanalyses, to Dr. G. Czira for providing some of the MS data, to A. Fürjes and I. Sinka for their NMR technical assistance and to OTKA (Hungarian Academic Research Found Grant K 68734) and to Richter Gedeon Rt for financial assistance.

\section{References}

1. (a) Guandolini, L.; Martini, E.; Gratteri, P.; Ghelardini, C.; Varani, K.; Romanelli, M. N. ARKIVOC 2006, (viii), 50. (b) Zhi, H.; Chen, L.-m.; Zhang, L.-1.; Liu, S.-j.; Wan, D. C. C.; Lin, H.-q.; Hu, C. Arkivoc 2008, (xiii), 266.

2. (a) Marco-Contelles, J.; do Carmo Carreiras, M.; Rodriguez, C.; Villarroya, M.; Garcia, A. G. Chem. Rev. 2006, 106, 116. (b) Czollner, L.; Treu, M.; Froehlich, J.; Kueenburg, B.; Jordis, U. ARKIVOC 2001, (i), 191.

3. Ishikawa, T.; Kudo, K.; Kuroyabu, K.; Uchida, S.; Kudoh, T.; Saito, S. J. Org. Chem. 2008, 73, 7498.

4. Sánchez, I. H.; Soria, J. J.; López, F. J.; Larraza, M. I.; Flores, H. J. J. Org. Chem. 1984, 49, 157.

5. Liang, P.-H.; Liu, J.-P.; Hsin, L.-W.; Cheng, C.-Y. Tetrahedron 2004, 60, 11655.

6. Fan, C.-A.; Tu, Y.-Q.; Song, Z.-L.; Zhang, E.; Shi, L.; Wang, M.; Wang, B.; Zhang, S.-Y. Org. Lett. 2004, 6, 4691. 
7. Malahowszki, W. P.; Paul, T.; Phounsavath, S. J. Org. Chem. 2007, 72, 6792.

8. (a) Kosley, R. W., Jr.; Davis, L.; Taberna, V. Eur. Pat. 0653427, 1995. (b) Davis, B. WO Pat. 00/33840, 2000.

9. Gorka, Á.; Hazai, L.; Szántay, Cs., Jr.; Háda, V.; Szabó, L.; Szántay, Cs. Heterocycles 2005, $65,1359$.

10. Johansson, A. M.; Mellin, C.; Hacksell, U. J. Org. Chem. 1986, 51, 5252.

11. Copinga, S.; Tepper, P. G.; Grol, C. J.; Horn, A. S.; Dubocovich, M. L. J. Med. Chem. 1993, 36, 2891.

12. Frigerio, M.; Santagostino, M.; Sputore, S. J. Org. Chem. 1999, 64, 4537.

13. (a) Nicolaou, K. C.; Zhong, Y.-L.; Baran, P. S. J. Am. Chem. Soc. 2000, 122, 7596. (b) Nicolaou, K. C.; Baran, P. S.; Zhong, Y.-L. J. Am. Chem. Soc. 2001, 123, 3183. (c) Nicolaou, K. C.; Montagnon, T.; Baran, P. S.; Zhong, Y.-L. J. Am. Chem. Soc. 2002, 124, 2245 . 